accommodating themselves to the unnatural conditions of town life, and the perpetual intermarriage of the survivors in this struggle. for existence, have had the effect of producing a race more or less immune to those deteriorating influences which prove so fatal by way of tuberculosis to the country-bred people from among whom our town populations are so constantly recruited. Others again have held that the comparative freedom of the Jews from tuberculosis is due to their (also comparative) freedom from alcoholism, and in support of this we may refer to what was said at the recent Congress of Tuberculosis by Professor Brouardel, who maintained that alcoholism was "the most potent factor in the propagation of tuberculosis." The whole problem is one of extreme interest. We will not take it upon ourselves to say even that it is a fact that tuberculosis does spare the Jew, or to what extent there is a due foundation for the general belief on the subject. What is evident, however, is that if there is any truth in this belief - a belief be it said which is vouched for by many well-known and highly accredited names-the problem of the prevention of consumption extends far beyond those mere details as to germs and infection with which 80 many people are now occupying themselves. The present Royal Commission which is investigating the possibility of tuberculosis infection passing from animals to men is engaged on a most interesting task ; but whatever conclusions it may arrive at they will not solve the problem.

\section{RUPTURE OF THE TENDO ACHILLIS.}

Mr. Lynn Thomas, C.B., F.R.C.S., urges the advantages of treating ruptured tendo achillis by early massage and by walking avout with the foot. held by a metal splint so arranged along the anterior aspect of the joint as to prevent its being bent so far as to stretch the reparative material but without extension of the foot. In doing physical drill on board ship on his way out to South Africa he had the misfortune to rupture his tendon, and he treated it in the way mentioned. HLaving had an aluminium spatula moulded to the shape of the bend of the ankle on its anterior aspect, this was pushed into a thick indiarubber tube, which not only acted as a padding but kept the splint in its place by preventing the splint from moving downwards under the boot-lace. On the boot being put on, this anterior splint was applied, extending from the middle of the dorsum of the foot to several inches above the ankle. On lacing up the boot the lace was made to pass round and round the splint in its lower few turns. In the upper part it passed entirely in front of it. The foot was thus held firmly at a right angle. Mr. Thomas thinks that by this line of treatment a stronger union is obtained than by either immediate suturing or the fixation of the limb by splints, because the call to provide sufficient reparative callus to bind the ruptured ends of the tendons is much greater during movement in the dependent position than it is during rest in an elevated or in a fixed position. The great difference in the quantity of reparative material so produced is well shown by a comparison of human and veterinary surgery in cases of broken bones and tendons. Mr. Lynn Thomas also points out a new sign of rupture of the tendo achillis. He says that much may be learned from the shape of the surface marking produced after a few days by the extravasated blood in the various injuries which occur in the neighbourhood of the ankle, and that when the tendo achillis is ruptured there is a bilateral discolouration on each side of the tendon running downwards below both malleoli and joined at the level of the malleoli, or the seat of the ruptured tendon, by a transverse band, thus giving to the bruise a characteristic $\mathrm{H}$-shape which is pathognomonic of a breach in the tendo achillis.

\section{HOW TO VACCINATE.}

Commenting on the remarks of Dr. Andrewes concerning susceptibility to re-vaccination, to which we referred last week, Dr. Albert Benthall ${ }^{1}$ states that he has recently re-vaccinated a rather larger number of persons-namely, 193 individuals, of whom 162 were males, and 31 were females. Out of the whole number of re-raccinations there was not one single failure, all the arms developing wellmarked vesicles in the three places scarified. Among these cases six had been recently vaccinated with negative result, but they showed no insusceptibility in his hands. In 72.53 per cent. of the cases the vesicles were large and well formed; in 16.06 per cent. they were moderate in size ; while in just under 11.4 per cent. they were small, but not under a quarter of an inch in diameter. The lymph used was that of the Jenner Institute. The arms were first washed with soap and warm water, and in the case of the men shaved; the region where vaccina tion was to take place was then well rubbed with hot boric acid solution (saturated), and afterwards dried with boric lint. The vaccine was placed on the skin in three places, and the epidermis was scratched through the vaccine with a blunt lancet; hardly any blood was perceptible. In all these cases and many others dealt with in the same manner, the re-vaccination took without undue inflammation or enlargement of glands. The point recommended for vaccination is the outer side of the left forearm. No shield and no lint is advised, the best results being obtained when the following directions are carried out: the undervest sleeve should be turned up and nothing worn under the sleeve of the white shirt which is always very loose. Where a coloured shirt is worn a piece of clean, soft, old cambric handkerchief should be tacked to the inside of the coloured sleeve. In this way the vesicles are hardly touched, and the arm is kept cool. The only application recommended is a liberal and frequent dusting with vinolia powder, and with these precautions it is found as a rule that the vesicles remain dry and unbroken. The lancet was sterilised with boiling water before each separate vaccination.

\footnotetext{
1 Lancet, Jan. 18.
}

\title{
PROGRESS IN ANeSTHETICS.
}

In reference to the causation of death produced under, or as the direct result of, anæsthesia, Mitchell Banks ${ }^{1}$ states that, in his opinion, the majority of

so-called cases of sudden collapse under anæsthetics are not sudden in reality, but danger has been threatening for some time before the collapse 